\title{
The Caudal Medial Entorhinal Cortex: a Selective Role in Recollection-Based Recognition Memory
}

\author{
Magdalena M. Sauvage, ${ }^{1,2}$ Zachery Beer, ${ }^{1,2}$ Muriel Ekovich, ${ }^{1}$ Lucy Ho, ${ }^{1}$ and Howard Eichenbaum ${ }^{1}$ \\ ${ }^{1}$ Center for Memory and Brain, Boston University, Boston, Massachusetts 02215, and ${ }^{2}$ Functional Architecture of Memory Unit, Mercator Research Group, \\ Faculty of Medecine, Ruhr University Bochum, Bochum 44 801, Germany
}

Recent studies have suggested that the caudal medial entorhinal cortex (cMEC) is specialized for path integration and spatial navigation. However, cMEC is part of a brain system that supports episodic memory for both spatial and nonspatial events, and so may play a role in memory function that goes beyond navigation. Here, we used receiver operating characteristic analysis to investigate the role of the cMEC in familiarity and recollection processes that underlie nonspatial recognition memory in rats. The results indicate that cMEC plays a critical and selective role in recollection-based performance, supporting the view that cMEC supports memory for the spatial and temporal context in which events occur.

\section{Introduction}

In 2004, Fyhn et al. reported the existence of neurons in the dorsocaudal medial entorhinal cortex (dcMEC) that fired when rats occupy multiple periodic spatial locations arranged in a gridlike pattern, leading the authors to describe them as "grid cells" (Hafting et al., 2005). Subsequent studies have also identified grid cells in the ventral part of the caudal MEC (Brun et al., 2008) as well as other characteristics of grid cells that have led some to suggest that caudal MEC (cMEC) may support spatial mapping and path integration (for review, see McNaughton et al., 2006; Moser et al., 2008). However, the cMEC is a part of the parahippocampal region (for a review, see van Strien et al., 2009), a major component of the medial temporal lobe memory system that supports episodic memory for both spatial and nonspatial information (for review, see Eichenbaum, 2000). Accordingly, the cMEC should also play an important role in nonspatial (as well as spatial) episodic memory.

Previous studies have examined the effects of damage to the entorhinal cortex, and these report deficits extending to both spatial and nonspatial memory. However, in these studies the damage was not restricted to the MEC. Thus, lesions including portions of both lateral and medial entorhinal cortex impair trace fear conditioning in rabbits (Esclassan et al., 2009), maze performance in rats (Steffenach et al., 2005), and nonspatial stimulus association in monkeys (Buckmaster et al., 2004). Other studies have reported involvement of the lateral entorhinal cortex (LEC) in nonspatial social recognition (Petrulis et al., 2005) and conditioned odor aversion (Ferry et al., 2006). These findings and the absence of grid cells in the LEC (Hargreaves et al., 2005) suggest

Received Aug. 17, 2010; revised Sept. 17, 2010; accepted Sept. 27, 2010.

This work was supported National Institute of Mental Health Grants MH52090 and MH71702. We thank Prof. Rebecca Burwell for her help with the flat map analysis technique and David Kim (Undergraduate Research Opportunities Program trainee) for assistance with behavioral training.

Correspondence should be addressed to Howard Eichenbaum, Center for Memory and Brain, 2 Cummington Street, Boston, MA 02215. E-mail: hbe@bu.edu.

DOI:10.1523/JNEUROSCI.4301-10.2010

Copyright $\odot 2010$ the authors $\quad$ 0270-6474/10/3015695-05\$15.00/0 involvement of the lateral entorhinal cortex in nonspatial memory, but leave open the question of whether damage restricted to MEC, and in particular to cMEC, would result in memory deficits that are restricted to spatial memory.

To address the important question of whether damage restricted to cMEC affects nonspatial memory, we asked whether selective cMEC lesions would result in a deficit in odor-cued episodic memory. For this purpose, we used a recognition memory task in which the contribution of episodic recall can be distinguished from mere familiarity with previously experienced stimuli by analysis of the receiver operating characteristic (ROC) function of recognition performance (for review, see Fortin et al., 2004; Eichenbaum et al., 2010; Sauvage, 2010). The results provide strong evidence that $\mathrm{cMEC}$ damage selectively eliminates the contribution of recollection to recognition memory, indicating a role for cMEC that extends to nonspatial memory.

\section{Materials and Methods}

Subjects and stimuli. Fourteen male Long-Evans rats (250-300 g) were maintained under reverse light/dark cycle (7:00 A.M. light off; 7:00 P.M. light on) at a minimum of $90 \%$ of normal body weight and tested in their home cage. The stimulus odors were common household scents (thyme, paprika, coriander, etc.) mixed with playground sand. The scented sand was contained in Nalgene plastic cups (one odor per cup) that were lowered on a platform in the front part of the cage. A pool of forty household odors was available; 20 were used each day.

Behavioral paradigm. Behavioral training, data collection, and ROC analyses followed the protocols described by Fortin et al. (2004). On each day, rats were presented with a unique list of odors (study list). After a delay, animals were tested for their ability to distinguish between the odors belonging to this list (old odors) and odors that were part of the pool but that did not belong to the list (new odors) (Fig. 1). The study list changed each day and odors were chosen in a pseudorandom manner. Rats first learned to dig in a cup filled with unscented sand to retrieve a buried reward (a quarter of a Froot Loop cereal piece). Subsequently, animals were trained on a nonmatching-to-sample rule. On each trial, rats initially sampled one stimulus. After the delay, they were presented with another stimulus that was identical or different from the sample. When the odor was not the same as the sample, the rat could retrieve a 
buried reward by digging in the test cup (nonmatch). Conversely, when the odor was the same as the sample, the rats could refrain digging and move to the back of the cage to receive a food reward (match). Training on this rule lasted $\sim 2$ months ( 20 trials a session, one session a day) and consisted of several steps in which the number of studied odors progressively increased from one to 10 , the memory delay from one to $30 \mathrm{~min}$, and the number of test odors from two to 20 (always half old, half new). Animals transitioned between training stages when a performance level of $80 \%$ correct for two consecutive days was reached (Fortin et al., 2004).

Data collection. When subjects reached criteria with a list of 10 study odors, 30 min delay, and 20 test odors per day, they were overtrained for a week. Subsequently, to generate ROC curves, which require measuring performance across different decision criteria, we varied the reward payoffs for correct old and new responses as well as the effort required in responding to the test stimuli. Five different combinations of cup sizes and amount of reward in the test cup and at the back of the cage were introduced to alter the subjects' bias in responding to, or withholding a response to the test stimuli (Fig. 2). Each bias level was used once a week in a pseudorandom manner. Performance stabilized after three sessions per bias ( 15 sessions total), after which data were collected to generate the preoperative ROC curves. Data were averaged over four sessions for each bias level, and the preoperative ROC curves were generated by plotting the probability of hits (correct recognition of an old odor) against the probability of false alarms (incorrect recognition of a new odor) across five response bias levels. Subsequently, animals were divided into two groups matched for overall performance; one group $(n=7)$ received bilateral NMDA lesions of the cMEC (Fig. 3), whereas the other group $(n=7)$ was sham-operated. After a two-week recovery, animals were retested and the postoperative ROC functions were generated in a similar manner as the preoperative curves.

ROC analysis. Groups and individual ROC data were analyzed using a standard least-squares method with $\mathrm{R}$ and $d^{\prime}$ as parameters (Yonelinas, 1994) and the best fitting ROC curve was generated using an excel solver routine (Yonelinas et al., 1998; for review, see Yonelinas and Parks, 2007). Briefly, the excel solver uses a model for recognition memory based on the probability of recollection and the distance between the familiarity distributions of new and old items over five bias levels. This model assumes that recognition involves two distinct processes, recollection $(R)$ and familiarity $(F)$, and can be illustrated by $P($ old $\mid$ old $)=R+$ $(1-R) F_{\text {old }}$, where $F_{\text {old }}=\Phi\left(d^{\prime} / 2-c_{\mathrm{i}}\right)$; and $P($ old $\mid$ new $)=F_{\text {new }}$, where $\mathrm{F}_{\text {new }}=\Phi\left(-d^{\prime} / 2-c_{\mathrm{i}}\right)$, in which $d^{\prime}$ is the distance between the equalvariance Gaussian strength distributions for old and new items, $c_{\mathrm{i}}$ the response criterion at point $i$, and $\Phi$ is the cumulative response function.

To study the shape of the ROC function (linear or curvilinear) in a model-independent manner, $z$-scores corresponding to the area under the normal distribution ( mean $=0 ; \mathrm{SD}=1$ ) were calculated for each hit and false alarm rate. These $z$-scores were fitted with regression functions to plot $z$-ROC functions and the quadratic coefficient of the functions was assessed and compared with zero.

To directly compare scores on recollection (probability estimate) and familiarity (the distance between the old and new familiarity distributions), $d^{\prime}$ scores of each subject were transformed into $F$ scores-probability estimates for familiarity. $F$ estimates were obtained by calculating the probability of a hit given a false alarm rate, chosen so that familiarity and recollection estimates were in the same range.

Statistics. Unpaired $t$ tests were used to compare between groups $R$ and $d^{\prime}$ individual values and paired $t$ tests to compare within-group individual values. A two-way ANOVA was performed with $R$ and $F$ as parameters.

Surgery. Lesions were performed under isoflurane anesthesia (1\%) by injecting $0.1 \mu \mathrm{l}$ of NMDA $(10 \mathrm{mg} / \mathrm{ml})$ in the cMEC in two sites per hemisphere with a Hamilton syringe driven by a micropump. Controls were sham-operated, with the syringe lowered in the cortex above the sites of injection. All procedures were approved by the Boston University Institutional Animal Use Committee.
Test list: 20 odors ( 10 old, 10 new)

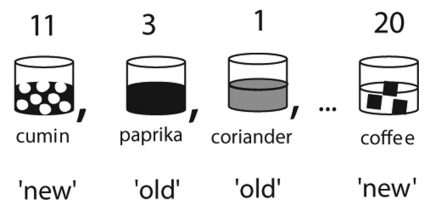

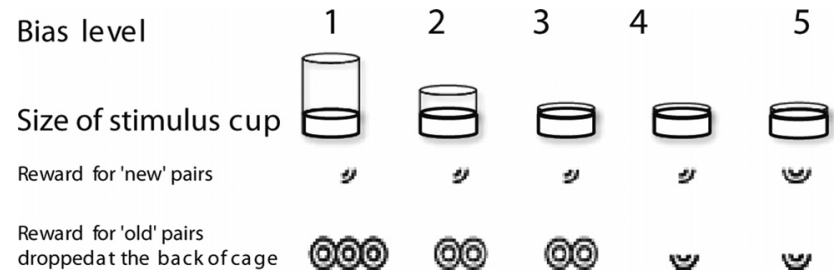

Figure 2. Test cup sizes and reward pay-offs for each bias level.

Extent and location of the lesions. To evaluate the extent of damage (Fig. $3 a$ ) as well as damage layer-by-layer within cMEC, surface measurements were performed on sagittal sections using the Axio-vision software from Zeiss. Dorsal and ventral borders of the cMEC were defined as described in the Watson and Paxinos rat brain atlas (1998). To ensure that the damage was restricted to the cMEC, unfolded maps of the cMEC were created following the procedure described by Dolorfo and Amaral (1998). Brains were cut in the sagittal plane, so the dorsal border of the cMEC was used as an alignment point (Fig. $3 b$, dashed line). Lesions were mapped onto the surface of layer II and unfolded onto straight lines perpendicular to the dorsal border of the cMEC. Because the lesions were circumscribed to the caudal part of the MEC, the unfolded map was restricted to anteroposterior levels -7 to -10.5 . Moreover, because sagittal sections were used and the lesions did not exceed the mediolateral levels 3.4 to 5 , the reconstructions are restricted to this coordinate range (Fig. $3 b$, yellow lines).

\section{Results}

Preoperative ROC curves were consistent with the pattern observed in item recognition performance in healthy humans and rats, and indicated the contribution of both recollection and familiarity to item recognition memory (Fig. 4a) (Yonelinas, 1994; Fortin et al., 2004). The preoperative ROC functions for groups that were assigned for later sham operation or cMEC lesions were asymmetric with positive $y$-intercepts, reflecting a significant contribution of recollection $\left(y\right.$-intercepts: $\operatorname{sham}_{\text {pre-op }}=0.49$ different from $0, t_{6}=19.22, p<0.001 ;$ cMEC $_{\text {pre-op }}=0.42, t_{6}=7.15$, $p<0.001$; confirmed by the slopes of the $z$-transformed regression ROC curves $<1, z$-ROC slopes: sham $_{\text {pre-op }}=0.52, t_{6}=-7.32$, $p<0.001 ; \mathrm{cMEC}_{\text {pre-op }}=0.68, t_{6}=-7.14, p<0.001$ ) (for review, see Yonelinas and Parks, 2007). Preoperative ROC functions for both groups were also curvilinear, reflecting a significant contribution of familiarity to recognition memory in this task ( sham $_{\text {pre-op: }}$ : $F_{\text {Quad 2,2 }}=19.52, p<0.050 ;$ cMEC $_{\text {pre-op }}: F_{\text {Quad 2,2 }}=24.20, p<0.050$ confirmed by a linear $z$-ROC curve: $z$-ROC quadratic coefficient not different from zero, sham ${ }_{\text {pre-op }}: t_{6}=-1.24, p>0.050, \mathrm{cMEC}_{\text {pre-op: }}$ : $t_{6}=0.22, p>0.050$ ) (for review, see Yonelinas and Parks, 2007), and a familiarity index $d^{\prime}$ different from 0 ( sham $_{\text {pre-op }}: d^{\prime}=0.77 \pm 0.06$, $t_{6}=13.88, p<0.001, \mathrm{cMEC}_{\text {preop }}: d^{\prime}=0.78 \pm 0.14, \mathrm{t}_{6}=5.68, p=$ $0.002)$. In addition, indices of recollection and familiarity $\left(d^{\prime}\right)$ did not differ significantly between groups before surgery $\left(R: t_{12}=1.37\right.$, $\left.p>0.050, d^{\prime}: t_{12}=-0.05, p>0.050\right)$.

Subsequently, cMEC lesions and sham surgeries were performed. Areal measurements revealed that an average of $68 \pm 3 \%$ 
a

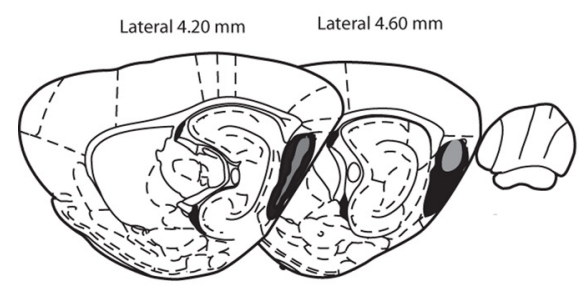

b

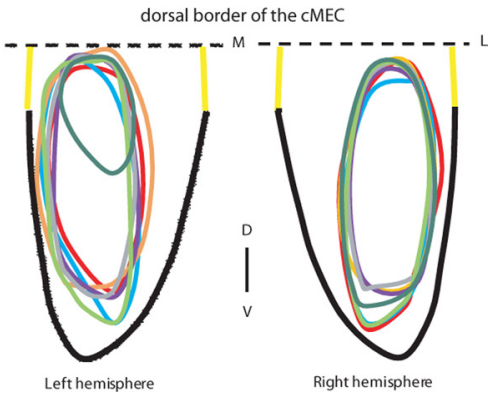

Figure 3. Extent and location of the CMEC lesions. $\boldsymbol{a}$, Area measurements. Gray, Smallest lesion; black, largest lesion. $\boldsymbol{b}$, Unfolding the CMEC revealed that damage was restricted to the CMEC. Seven individual lesions are represented. The dashed black line represents the dorsal border of the CMEC and the thick continuous black line represents the ventral border of the CMEC, according the rat brain atlas of Watson and Paxinos. The unfolded map is restricted to the mediolateral levels $\pm 3.4-5$ (yellow lines) and the anteroposterior levels -7.5 to -10 . D-V, Dorsoventral axis; $L-M-L$, mediolateral axis.

by a linear $z$-ROC ( $z$-ROC quadratic coefficient not different from zero: $t_{6}=-0.14$, $p>0.050)$ and a familiarity index $d^{\prime}$ different from $0\left(d^{\prime}=0.99 \pm 0.13, p<0.001\right)$ (Fig. 2b). The selective effect of cMEC damage on recollection was confirmed by a decreased postoperative recollection estimate compared with the preoperative estimate for the same animals (cMEC: $R_{\text {pre-op }}=0.37$, $\left.R_{\text {post-op }}=0.11, t_{6}=8.60, p<0.001\right)$. By contrast, damage to the cMEC did not significantly alter the familiarity estimate compared with preoperative performance (cMEC: $d_{\text {pre-op }}^{\prime}=0.78, d_{\text {post-op }}^{\prime}=0.99$, $\left.t_{6}=-1.80, p>0.050\right)$.

Furthermore, direct between-group comparisons of the recollection index and

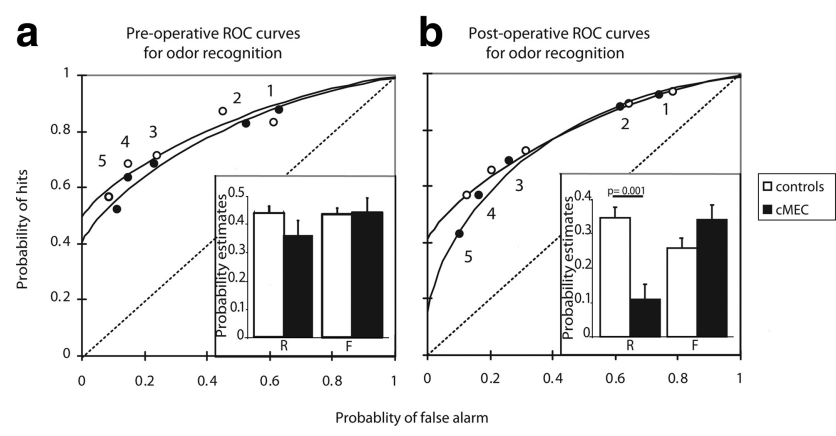

Figure 4. ROC curves and recollection and familiarity indices for odor recognition memory. $\boldsymbol{a}$, Standard ROC functions for item recognition. Both preoperative groups used recollection and familiarity to solve the task, as reflected by asymmetrical and curvilinear ROC curves, and recollection and familiarity indices did not significantly differ between preoperative groups (insert). $\boldsymbol{b}$, Rats with MEC damage were impaired in recollection, as shown by a decrease of the $y$-intercept and a drop of the recollection index (insert), whereas familiarity remained unaffected and performance of controls was unchanged following sham surgery. Error bars represent SEM; Controls, Sham-operated rats; MEC, rats with MEC lesions.

of the cMEC was damaged. Layer analyses indicated that layers 2 , $3 / 4$, and 5 had the greatest damage $(71 \%, 75 \pm 6 \%$, and $60 \pm$ $11 \%$, respectively), whereas layers 1 and 6 were less damaged ( $39 \pm 7 \%$ and $37 \pm 8 \%$, respectively). There was no obvious damage outside the borders of cMEC.

Postoperatively, the sham surgery did not significantly alter the ROC function. In sham-operated animals, the ROC curve continued to be asymmetric $(y$-intercept $=0.42$ different from 0 , $p<0.001$ ) (Fig. 4b), confirmed by the slope of the sham $z$-ROC curve $<1(z$-ROC slope $=0.71, p=0.010)$. The ROC curve for sham-operated animals also continued to be significantly curvilinear $\left(F_{\text {Quad 2,2 }}=463.24, p<0.050\right)$, confirmed by a linear $z$-ROC curve $(z$-ROC quadratic coefficient not different from zero, $\left.t_{6}=-0.14, p>0.050\right)$ and a familiarity index $d^{\prime}$ different from $0\left(d^{\prime}=0.75 \pm 0.08, p<0.001\right)$. In addition, preoperative and postoperative recollection and familiarity estimates did not significantly differ for sham-operated animals (sham: $R_{\text {pre-op }}=$ $0.44, R_{\text {post-op }}=0.35, t_{6}=2.38, p>0.050 ; d_{\text {pre-op }}^{\prime}=0.77, d_{\text {post-op }}^{\prime}=$ $\left.0.75, t_{6}=0.36, p>0.050\right)$.

In striking contrast to the sham-operated group, damage to the cMEC severely reduced the contribution of recollection to recognition memory (Fig. $4 b$ ). In cMEC rats, the $y$-intercept of the ROC curve was no longer significantly different from 0 ( $y$ intercept $=0.15, p>0.050$ ), whereas the ROC function was still significantly curvilinear $\left(F_{\text {Quad 2,2 }}=67.35, p<0.050\right)$, confirmed the normalized familiarity estimate (see Material and Methods), which allows direct comparison to the $R$ index, confirmed that damage to the cMEC selectively affected recollection (interaction: $F_{(1,24)}=18.66, p<0.001$; post hoc $R: p=0.001 ; F: p>$ 0.050 ). Correspondingly, the postoperative recollection index for cMEC rats was significantly lower than that of sham-operated rats (sham: $R=0.35 \pm 0.03$; cMEC: $R=0.11 \pm 0.05 ; t_{12}=$ $-4.39, p=0.001$ ), whereas the familiarity estimate did not significantly differ between groups postoperatively $\left(d^{\prime}{ }_{\text {sham }}=0.75 \pm\right.$ $\left.0.08 ; d^{\prime}{ }_{\text {MMEC }}=0.99 \pm 0.13 ; t_{12}=-1.68, p>0.050\right)$.

In addition, the impairment in the cMEC group could be attributed to a decrease in remembered items, specifically at the bias level normally associated with strong recollection (bias 5; hit $_{\text {sham }}=0.60$, hit $_{\text {cMEC }}=0.46, t_{12}=-3.35, p=0.006$ ), with no significant difference in false alarms $\left(\mathrm{fa}_{\text {sham }}=0.13, \mathrm{fa}_{\mathrm{cMEC}}=\right.$ $\left.0.10, t_{12}=-0.47, p>0.050\right)$. This finding indicates a reduction in memory for previously experienced stimuli without a change in response bias.

Finally, we analyzed the data using an alternate model of recognition memory, the unequal variance signal detection model, which suggests that the asymmetry of the ROC curve results from the combination of two parameters: a difference in memory strength between the distributions of old and new items and a larger variance of the distribution of old items compared with that of the new items $\left(V_{\text {old }}>V_{\text {new }}=1\right)$ (for review, see Wixted, 2007). Data analysis showed that damage to the cMEC selectively eliminates one parameter of the ROC function, specifically the inequality of variances between old and new items (cMEC: $V_{\text {old }}=$ 0.98 not different from $\left.V_{\text {new }}=1.0, t_{6}=-0.12, p>0.050\right)$. By contrast, there was no change in the other parameter of the ROC function, $d^{\prime}$, which reflects the difference in memory strength between old and new items $(p>0.050)$. In summary then, the single process model confirms that cMEC lesion selectively eliminates one parameter supporting the identification of old items, which is comparable to altering the recollection process in the dual-process theory, leaving intact another parameter comparable to familiarity.

\section{Discussion}

These results provide the first evidence that the cMEC is involved in recognition memory in a task devoid of a spatial component, suggesting a broader role in memory function than contributing solely to spatial cognition. In addition, the present data show that the cMEC contributes specifically to recollection-based memory and not to a sense of familiarity for recently experienced stimuli. Importantly, the conclusion that cMEC plays a broader role in 
memory does not rest on the dual-process theory, which is contentious (Wixted, 2007); viewed from the unequal variance signal detection model, the data show that cMEC plays a role in strong nonspatial memories, although that model does not suggest any manipulation that should equalize the variance of the old and new distributions while leaving the strength parameter unchanged.

These findings are in agreement with a broad view of cMEC function in spatial representation that goes beyond navigation and path integration, consistent with recent neurophysiological evidence. Thus, in a recent study where rats performed a working memory task in which they alternated between left and right turns on a T maze, neurons in dcMEC fired when rats traversed multiple spatial locations, similar to the grid cell pattern (Lipton et al., 2007). In addition, dcMEC cells fired differentially on leftand right-turn trials, even in sections of the maze that were traversed on both trial types. By contrast, hippocampal neurons better predicted the animal's location on the stem and more poorly predicted which type of episode was ongoing. Thus, one can characterize cMEC neurons as encoding the spatial and temporal context of the current location whereas hippocampal neurons better encode the location of an event within a particular context (Lipton and Eichenbaum, 2008). In the recognition task used in the current study, the cMEC may have contributed to recollection by providing information about the spatial and temporal context of the studied odors. In our recognition paradigm, temporal context is especially important because the odor stimuli are reused across days, so a key demand is to remember which odors were experienced in the study phase of the current session as opposed to previous sessions.

Parallel findings on representation of spatial and temporal context were described for the adjacent and highly interconnected parahippocampal cortex in humans, which is commonly activated by viewing spatial environments (Epstein and Kanwisher, 1998). In further studies on this area, Bar et al. (2008) found that the parahippocampal cortex was also activated selectively when subjects viewed common objects that evoke rich spatial contextual associations (e.g., a refrigerator evokes the association of a kitchen), as contrasted with objects that lack strong contextual associations (e.g., a pencil). Furthermore, an overlapping area of the parahippocampal cortex was activated when subjects viewed objects that had strong temporal associations (Aminoff et al., 2007). Thus, one can characterize the parahippocampal cortex, which is intimately connected with MEC, as representing the spatial and temporal context of remembered objects.

These findings support a hypothesis about the functional organization of the medial temporal lobe (Eichenbaum et al., 2007). According to this view, the perirhinal cortex and closely connected lateral entorhinal cortex process information about the familiarity of individual items whereas the parahippocampal cortex and closely connected MEC represent the spatial and temporal context in which important events occur. Subsequently, outputs of these areas converge within the hippocampus where conjunctions of item and context information are represented. Consistent with this view, a recent study reported the robust development of neuronal firing patterns that encode particular objects in distinct contexts as animals learn the differential significance of the items in different contexts (Komorowski et al., 2009). Correspondingly, the hippocampus is critical to remembering items in the spatial and temporal context in which they were experienced (Ergorul and Eichenbaum, 2004), and for recollection in recognition memory (Fortin et al., 2004), particularly when animals must remember the context in which odors were presented (Sauvage et al., 2008).

Furthermore, in support of the hypothesis that the cMEC provides information required for recollection-based judgments, the magnitude of the recollection deficit observed following cMEC lesions was similar to, albeit less dramatic than, that observed following hippocampal lesions using the same behavioral paradigm. Thus, comparing the ROC recollection indices between control and different lesion groups, the difference between the $R$ index for the sham-operated group and that of the cMEC group in the present study was 0.25 , slightly lower than the difference in $R$ between the sham control and hippocampal groups in the Fortin et al. (2004) study (0.34). Importantly, recollection was selectively impaired in both cases and the impairment resulted from a decrease in the proportion of hits (correctly remembered items) and no change in the proportion of false alarms (Fortin et al., 2004). In contrast, the recollection deficit reported following lesion of the medial prefrontal cortex was of the same magnitude $\left(R_{\text {sham }}-R_{\mathrm{mPFC}}=0.25\right)$ as observed here, but resulted from an increase in false alarms (increased identification of a new odor as being old) with no change in the hit rate, suggesting that the medial prefrontal cortex and medial temporal areas contribute differently to the recollection process (Farovik et al., 2008).

The current results add important evidence supporting the proposed functional organization of the medial temporal lobe, showing that $\mathrm{CMEC}$ is not involved in familiarity for specific items but is a part of the circuitry that is essential to recollection, which depends on memory for the spatial and temporal context in which events occur (Eichenbaum et al., 2007). This perspective is consistent with the observations on cMEC in spatial representation, but suggests a broader role in representation of spatial and temporal context of importance to episodic memory.

\section{References}

Aminoff E, Gronau N, Bar M (2007) The parahippocampal cortex mediates spatial and nonspatial associations. Cereb Cortex 17:1493-1503.

Bar M, Aminoff E, Schacter DL (2008) Scenes unseen: the parahippocampal cortex intrinsically subserves contextual associations, not scenes or places per se. J Neurosci 28:8539-8544.

Brun VH, Solstad T, Kjelstrup KB, Fyhn M, Witter MP, Moser EI, Moser MB (2008) Progressive increase in grid scale from dorsal to ventral medial entorhinal cortex. Hippocampus 18:1200-1212.

Buckmaster CA, Eichenbaum H, Amaral DG, Suzuki WA, Rapp PR (2004) Entorhinal cortex lesions disrupt the relational organization of memory in monkeys. J Neurosci 24:9811-9825.

Dolorfo CL, Amaral DG (1998) Entorhinal cortex of the rat: topographic organization of the cells of origin of the perforant path projection to the dentate gyrus. J Comp Neurol 398:25-48.

Eichenbaum H (2000) A cortical-hippocampal system for declarative memory. Nat Rev Neurosci 1:41-50.

Eichenbaum H, Yonelinas AP, Ranganath C (2007) The medial temporal lobe and recognition memory. Annu Rev Neurosci 30:123-152.

Eichenbaum H, Fortin N, Sauvage M, Robitsek RJ, Farovik A (2010) An animal model of amnesia that uses Receiver Operating Characteristics (ROC) analysis to distinguish recollection from familiarity deficits in recognition memory. Neuropsychologia 48:2281-2289.

Epstein R, Kanwisher N (1998) A cortical representation of the local visual environment. Nature 392:598-601.

Ergorul C, Eichenbaum H (2004) The hippocampus and memory for "what," "where," and "when". Learn Mem 11:397-405.

Esclassan F, Coutureau E, Di Scala G, Marchand AR (2009) A cholinergicdependent role for the entorhinal cortex in trace fear conditioning. J Neurosci 29:8087-8093.

Farovik A, Dupont LM, Arce M, Eichenbaum H (2008) Medial prefrontal cortex supports recollection, but not familiarity, in the rat. J Neurosci 28:13428-13434.

Ferry B, Ferreira G, Traissard N, Majchrzak M (2006) Selective involvement 
of the lateral entorhinal cortex in the control of the olfactory memory trace during conditioned odor aversion in the rat. Behav Neurosci 120:1180-1186.

Fortin NJ, Wright SP, Eichenbaum H (2004) Recollection-like memory retrieval in rats is dependent on the hippocampus. Nature 431:188-191.

Fyhn M, Molden S, Witter MP, Moser EI, Moser MB (2004) Spatial representation in the entorhinal cortex. Science 305:1258-1264.

Hafting T, Fyhn M, Molden S, Moser MB, Moser EI (2005) Microstructure of a spatial map in the entorhinal cortex. Nature 436:801-806.

Hargreaves EL, Rao G, Lee I, Knierim JJ (2005) Major dissociation between medial and lateral entorhinal input to dorsal hippocampus. Science 308:1792-1794.

Komorowski RW, Manns JR, Eichenbaum H (2009) Robust conjunctive item-place coding by hippocampal neurons parallels learning what happens where. J Neurosci 29:9918-9929.

Lipton PA, Eichenbaum H (2008) Complimentary roles of hippocampus and medial entorhinal cortex in episodic memory. Neural Plast 2008:258467.

Lipton PA, White JA, Eichenbaum H (2007) Disambiguation of overlapping experiences by neurons in the medial entorhinal cortex. J Neurosci 27:5787-5795.

McNaughton BL, Battaglia FP, Jensen O, Moser EI, Moser MB (2006) Path integration and the neural basis of the 'cognitive map'. Nat Rev Neurosci 7:663-678.

Moser EI, Kropff E, Moser M-B (2008) Place cells, grid cells, and the brain's spatial representation system. Annu Rev Neurosci 31:69-89.

Paxinos G, Watson CR (1998) The rat brain in stereotaxic coordinates, Fourth Edition. New York: Academic.
Petrulis A, Alvarez P, Eichenbaum H (2005) Neural correlates of social odor recognition and the representation of individual distinctive social odors within entorhinal cortex and ventral subiculum. Neuroscience 130:259-274.

Sauvage MM (2010) ROC in animals: uncovering the neural substrates of recollection and familiarity in episodic recognition memory. Conscious Cogn 19:816-828.

Sauvage MM, Fortin NJ, Owens CB, Yonelinas AP, Eichenbaum H (2008) Recognition memory: opposite effects of hippocampal damage on recollection and familiarity. Nat Neurosci 11:8-16.

Steffenach HA, Witter M, Moser MB, Moser EI (2005) Spatial memory in the rat requires the dorsolateral band of the entorhinal cortex. Neuron 45:301-313.

van Strien NM, Cappaert NL, Witter MP (2009) The anatomy of memory: an interactive overview of the parahippocampal-hippocampal network. Nat Rev Neurosci 10:272-282.

Wixted JT (2007) Dual-process theory and signal-detection theory of recognition memory. Psychol Rev 114:152-176.

Yonelinas AP (1994) Receiver-operating characteristics in recognition memory: evidence for a dual-process model. J Exp Psychol Learn Mem Cogn 20:1341-1354.

Yonelinas AP, Parks CM (2007) Receiver operating characteristics (ROCs) in recognition memory: a review. Psychol Bull 133:800-832.

Yonelinas AP, Kroll NE, Dobbins I, Lazzara M, Knight RT (1998) Recollection and familiarity deficits in amnesia: convergence of remember-know, process dissociation, and receiver operating characteristic data. Neuropsychology 12:323-339. 